\title{
EI Niño-Oscilación del Sur, fase Modoki, y sus efectos en la variabilidad espacio-temporal de la precipitación en Colombia
}

\author{
(D) Estefanía Navarro-Monterroza, (D) Paola A. Arias*, (D) Sara C. Vieira \\ Grupo de Ingeniería y Gestión Ambiental (GIGA), Facultad de Ingeniería, Universidad de Antioquia, Medellín, Colombia
}

\begin{abstract}
Resumen
Se analizó la posible influencia del fenómeno de El Niño-Oscilación del Sur (ENOS) en la variabilidad espaciotemporal de la precipitación en Colombia a partir de registros de estaciones in situ durante el período 1970-2015 y de datos provenientes de registros satelitales disponibles a partir de 1998. Mediante los índices de temperatura superficial del mar (TSM) asociados al fenómeno ENOS, se evaluaron las anomalías en la precipitación por trimestre para cada tipo de evento, así como las correlaciones mensuales simultáneas y rezagadas entre cada índice y las anomalías en la precipitación. Con base en la información satelital se concluyó que en gran parte del país hubo una mayor precipitación durante los eventos de La Niña Modoki que durante La Niña Canónica en el trimestre de diciembre, enero y febrero, en tanto que la precipitación disminuyó más en la fase canónica de El Niño que en la fase Modoki. La significación estadística de las correlaciones rezagadas evidenció la influencia de la TSM del Pacífico este en las anomalías en la precipitación en Colombia con un rezago de 0 hasta 6 meses. Los resultados de este trabajo aportaron una mayor claridad sobre las variaciones de la distribución de la precipitación y la disponibilidad de agua en Colombia durante las diversas fases del fenómeno de ENOS, lo cual contribuye a la búsqueda de un manejo más eficiente del recurso hídrico. (C) 2019. Acad. Colomb. Cienc. Ex. Fis. Nat.
\end{abstract}

Palabras clave: ENOS; Modoki; Variabilidad espacio-temporal; Precipitación; Colombia.

EI Niño/Southern Oscillation Modoki and its effects on the spatiotemporal variability of precipitation in Colombia

\begin{abstract}
The possible influence of the El Niño-Southern Oscillation (ENSO) phenomenon on the spatiotemporal variability of the precipitation in Colombia is analyzed considering precipitation records from rain gauge dataset during the period 1970-2015, as well as records from satellite datasets available for the period 1998 to present. Based on sea surface temperature indexes associated with the ENSO phenomenon, we evaluated seasonal rainfall anomalies for each type of ENSO event, as well as monthly simultaneous and lagged correlations between ENSO indexes and precipitation anomalies. Results from the satellite data showed that there was more precipitation during La Niña Modoki than during the Canonical La Niña events in most of the country for the December-February period, while there was a larger reduction of precipitation during the canonical events of El Niño than the Modoki events. The statistical significance of lagged correlations showed that there was an influence of the surface temperature anomalies over the eastern Pacific region on precipitation in Colombia for a lag of 0 up to 6 months. This paper provides new insights about variations of precipitation distribution and water availability in Colombia during the different phases of ENSO, which is important for the efficient management of water resources in the region. (C) 2019. Acad. Colomb. Cienc. Ex. Fis. Nat.
\end{abstract}

Key words: ENSO; Modoki; Spatiotemporal variability; Precipitation; Colombia.

\section{Introducción}

El Niño-Oscilación del Sur (ENOS) es un modo de variabilidad interanual de la temperatura superficial del mar (TSM) en el océano Pacífico tropical que se caracteriza por generar claras anomalías climáticas en diferentes regiones del planeta (Rasmusson \& Carpenter, 1982). Inicialmente, se denominó El Niño al período durante el cual se presentan anomalías positivas de temperatura (calentamiento) en el este del Pacífico ecuatorial asociadas con un debilitamiento de los vientos alisios (Wyrtki, 1975); por su parte, La Niña (LN) se refiere al período de anomalías negativas de la temperatura (enfriamiento) asociadas a altas presiones en el Pacífico este tropical y a un fortalecimiento de los vientos alisios, es decir lo contrario de lo que ocurre durante los

\footnotetext{
*Correspondencia:

Paola A. Arias; paola.arias@udea.edu.co

Recibido: 27 de julio de 2018

Aceptado: 5 de marzo de 2019

Editor: José Pabón Caicedo
} 
eventos de El Niño (Philander, 1990; Mcphaden, et al., 1998). Así pues, en los estudios se han establecido dos fases de este fenómeno generadas por las interacciones entre el océano y la atmósfera a las cuales se hace referencia como eventos canónicos.

En estudios recientes se han detectado variaciones de los eventos de El Niño y la La Niña caracterizadas por un patrón de anomalías de la TSM localizadas en el centro del Pacífico tropical y no en el este del océano, como ocurre en las fases canónicas del ENOS, eventos que se conocen con el nombre Modoki. El Niño Modoki se caracteriza por la aparición de anomalías positivas en la TSM en el centro del Pacífico ecuatorial y de anomalías negativas en el este y el oeste del océano (Ashok, et al., 2007); por su parte, La Niña Modoki es la fase opuesta, y se caracteriza por una distribución de anomalías de TSM negativas y positivas en estas regiones, respectivamente, contrarias a las que ocurren durante El Niño Modoki (Ashok, et al., 2009). Estos eventos, producto de procesos naturales, afectan los esquemas de circulación atmosférica y de variabilidad climática en todo el planeta.

El fenómeno ENOS tiene un impacto significativo a nivel mundial que varía según la región y la temporada; por ejemplo, en Australia tiende a presentarse una época de sequía durante el trimestre de septiembre a noviembre con la aparición de eventos de El Niño, y la magnitud de sus efectos en la hidrología de esa región dependen de qué tan al extremo oriental del océano Pacífico tropical se localicen las anomalías en la TSM (Wang \& Hendon, 2007). Por otro lado, en el oeste de Estados Unidos se ha evidenciado una mayor frecuencia de sequías y altas temperaturas durante el trimestre de diciembre a febrero, las cuales pueden estar asociadas con El Niño Modoki y con su intensidad (Weng, et al., 2007). En otro estudio, Ropelewski \& Halpert (1996) evaluaron la relación entre los episodios fríos y cálidos del fenómeno ENOS y la distribución de la precipitación en diferentes regiones del mundo, y señalaron que en África la relación entre las anomalías de la TSM del Pacífico ecuatorial y la precipitación es poco significativa durante los eventos de La Niña, en tanto que durante El Niño se experimentan condiciones de sequía en el sureste del continente. Los cambios generados durante los diferentes eventos de ENOS en la climatología global son evidentes, por lo que la investigación de estos temas es fundamental para una mejor comprensión de su comportamiento y de su impacto a nivel local.

En Suramérica se han hecho diversos estudios sobre el impacto de los diferentes eventos del fénomeno de ENOS en el régimen de precipitación. Entre ellos se encuentra el de Grimm, et al. (2000), en el que se analizan las anomalías en la precipitación y en la circulación debidas a los eventos de El Niño y La Niña. Los autores encontraron que el sur de Brasil era la región que sufría el impacto más fuerte en la precipitación durante los episodios de El Niño, en tanto que en algunas áreas del este de los Andes en el sur de Suramérica se observaron anomalías positivas en la precipitación durante el trimestre de septiembre a noviembre. Por su parte, Silva (2012) encontró que en este mismo trimestre El Niño Modoki generaba anomalías positivas de lluvia al sur del sureste de Suramérica asociadas con un ciclón barotrópico en el Pacífico central ecuatorial. En los estudios de Tedeschi, et al. (2012, 2016a, 2016b) se analizó la influencia de los episodios canónicos y Modoki del ENOS y de sus eventos extremos en el patrón espacial de la precipitación en Suramérica. Estos trabajos demuestran que es fundamental tener en cuenta tanto la fase canónica como la Modoki del ENOS cuando se estudia la variabilidad de la precipitación para un mejor monitoreo de las condiciones hidrológicas en una región.

En Colombia, Poveda, et al. (1998) analizaron la influencia de los eventos de El Niño y La Niña en la variabilidad interanual de la hidroclimatología local y encontraron que la correlación entre las anomalías de la TSM en el océano Pacífico este ecuatorial y la precipitación era más fuerte durante el trimestre de diciembre a febrero $\mathrm{y}$, en algunos casos, durante junio, julio y agosto, en tanto que la correlación se debilitaba en el período de marzo, abril y mayo. Asimismo, Córdoba-Machado, et al. (2015) estudiaron la influencia del fenómeno ENOS en el régimen de precipitación en Colombia y observaron que las áreas del norte, el centro y el occidente del país experimentaban una reducción de la precipitación durante los eventos de El Niño en los trimestres de diciembre a febrero, junio a agosto y septiembre a noviembre, en tanto que en otras localidades ocurrían anomalías positivas en el régimen de precipitación durante estos períodos. Los autores también señalaron que durante los episodios de El Niño Modoki había una reducción de los valores de la precipitación en el suroccidente de Colombia durante el trimestre de septiembre a noviembre, en tanto que observaron un comportamiento contrario durante los eventos de El Niño, ya que en este periodo se registraron anomalías positivas en la precipitación.

Dada la clara influencia que cada uno de los episodios del fenómeno de ENOS ejerce en el régimen de precipitación de Colombia y la necesidad de mejorar la predicción del impacto futuro de estos eventos en la hidrología, en el presente estudio se analizó el impacto de los episodios canónicos y Modoki del fenómeno ENOS en el régimen de precipitación en el país durante los cuatro trimestres del año a partir de registros in situ y satelitales de precipitación, con el fin aportar al conocimiento de los efectos de los diferentes episodios de este fenómeno en distintas regiones del país.

\section{Materiales y métodos}

Datos. Se emplearon tres bases de datos para el análisis de la variabilidad espacio-temporal del régimen de precipitación en Colombia durante los eventos de El Niño, El Niño Modoki, La Niña y La Niña Modoki. El primer conjunto de datos incluye los registros diarios de la TSM provenientes de la reconstrucción extendida de la TSM (extended reconstructed sea surface temperatura, ERSST), versión 
4, de la National Oceanic and Atmospheric Administration (NOAA) desde 1854 hasta el presente, con una resolución de $2^{\circ}$ de latitud y $2^{\circ}$ de longitud. Con esta primera base de datos se clasificaron los trimestres de incidencia de cada uno de los eventos ENOS para el período de 1970 a 2015 a partir del índice del Niño 3 para el caso de los eventos canónicos y del índice El Niño Modoki Index (EMI) para los episodios en la fase Modoki. Además, se calcularon los índices del Niño 3.4, el Oceanic Niño Index (ONI) y el Niño 4.

La segunda base de datos se obtuvo de la versión 7 del Análisis de Precipitación Multisatelital de la Misión de Medición de Lluvias Tropicales (Tropical Rainfall Measuring Mission, TRMM), la cual corresponde a registros de la precipitación medida en $\mathrm{mm} / \mathrm{hr}$ desde 1998 hasta el presente, con una resolución de $0,25^{\circ}$ x $0,25^{\circ}$. Estos datos satelitales de la precipitación se tomaron para la celda $65^{\circ}-85^{\circ} \mathrm{O}$ y $5^{\circ} \mathrm{S}-15^{\circ} \mathrm{N}$. En los análisis de este trabajo también se consideraron los registros de precipitación in situ del Instituto de Hidrología, Meteorología y Estudios Ambientales de Colombia (IDEAM) durante el período de 1970 a 2015, los cuales se expresan en mm/mes.

Clasificación de los trimestres de presencia de los eventos de ENOS. Para determinar los trimestres según la presencia de El Niño y La Niña, se calcularon las anomalías normalizadas en la región del Niño 3, como lo proponen Kim, et al. (2009). A partir del valor de las anomalías calculadas por trimestre durante el período 1970 a 2015, se clasificaron los períodos de incidencia de El Niño durante los cuales se presentaron valores de anomalías normalizadas iguales o superiores a 1,0 y como trimestres de La Niña aquellos en los cuales el valor había sido igual o inferior a $-1,0$.

Para clasificar los trimestres de presencia de eventos de El Niño Modoki y La Niña Modoki se usó el EMI descrito en la ecuación 1. Este índice tiene en cuenta las anomalías normalizadas de la TSM en tres regiones ubicadas en el centro, este y oeste del océano Pacífico tropical. El EMI fue introducido por Ashok, et al. (2007) para determinar los períodos en los cuales ocurre este nuevo modo de acoplamiento entre océano y atmósfera dada su importancia en la variabilidad climática del planeta.

$$
E M I=[S S T A]_{A}-0,5[S S T A]_{B}-0,5[S S T A]_{C}
$$

Las variables entre corchetes representan las anomalías de la TSM en cada región; de este modo, la región A está definida entre $\operatorname{los} 165^{\circ} \mathrm{E}$ y $\operatorname{los} 140^{\circ} \mathrm{O}$ y entre los $10^{\circ} \mathrm{S} y$ $\operatorname{los} 10^{\circ} \mathrm{N}$; la región $\mathrm{B}$, entre $\operatorname{los} 110^{\circ} \mathrm{y} \operatorname{los} 70^{\circ} \mathrm{O}$ y $\operatorname{los} 15^{\circ} \mathrm{S} \mathrm{y}$ $\operatorname{los} 5^{\circ} \mathrm{N}$, y la región $\mathrm{C}$, entre los $125^{\circ} \mathrm{y} \operatorname{los} 145^{\circ} \mathrm{E}$ y los $10^{\circ} \mathrm{S} y$ $\operatorname{los} 20^{\circ} \mathrm{N}$. En estas áreas se calcularon las anomalías en cada trimestre para el período de 1970 a 2015. A partir de esto, se calculó el EMI con base en la ecuación 1 y se determinaron como trimestres de presencia de El Niño Modoki aquellos en los cuales los valores del EMI fueron iguales o superiores a 0,7 y comos trimestres de La Niña Modoki aquellos en que los valores del índice fueron iguales o inferiores a -0,7, como lo sugieren Ashok, et al. (2007).
Además de calcular los índices del Niño 3 y el EMI, en el estudio se usaron los índices del Niño 3.4 y el ONI, los cuales caracterizan los eventos canónicos, y el índice del Niño 4, que determina los eventos Modoki. El ONI se basa en las anomalías de la TSM en la región del Niño 3.4 , las cuales deben ser superiores a $0,5^{\circ} \mathrm{C}$ durante tres meses consecutivos en el caso de los eventos de El Niño, e inferiores a $-0,5^{\circ} \mathrm{C}$ para clasificarlos como eventos de $\mathrm{La}$ Niña. Este índice se obtuvo de la base de datos de la NOAA disponible en http:/Www.cpc.ncep.noaa.gov/products/ analysis_monitoring/ensostuff/ensoyears.shtml. Además, se calcularon los índices del Niño 3.4 y el Niño 4 con base en la metodología de Kim, et al. (2009), los cuales se definen a partir de las anomalías normalizadas de la TSM en la región del Niño 3.4 y el Niño 4, respectivamente. Los trimestres en los cuales el índice del Niño 3.4 fue superior a 1,0 se determinaron como aquellos con eventos de El Niño, en tanto que en los trimestres en los cuales el índice fue inferior a -1,0 se establecieron como lo que presentaban eventos de La Niña. Los trimestres en los cuales el índice del Niño 4 tuvo valores superiores a 1,0 se establecieron como aquellos con presencia de episodios de El Niño Modoki, en tanto que en la región del Niño 3 las anomalías estuvieron por debajo de ese valor; por último, los trimestres con eventos de La Niña Modoki se establecieron como aquellos en los cuales las anomalías normalizadas de la TSM en la región del Niño 4 estuvieron por debajo de -1 , mientras que en la región del Niño 3 estuvieron por encima de ese valor.

Selección de las estaciones del IDEAM. Se hizo un análisis de la calidad de la información en los registros de precipitación de las estaciones del IDEAM correspondientes al periodo de 1970 a 2015 con lo cual se depuraron los datos de todas las estaciones con registros desde 1970 o anteriores, y se seleccionaron aquellas que contaban con el $95 \%$ de los datos durante el periodo de 1970 a 2015 . A partir de este análisis, se seleccionaron las 48 estaciones que presentaban un $5 \%$ o menos de datos faltantes, con el fin de que al procesar la información y hacerle control de calidad, los resultados no se vieran alterados por la ausencia de datos. En la Tabla 1 se presenta la descripción de cada estación.

Anomalías en la precipitación. Se obtuvieron los mapas con el promedio de las anomalías de precipitación para cada evento (El Niño, El Niño Modoki, La Niña y La Niña Modoki) teniendo en cuenta los trimestres clasificados; además, se obtuvieron los mapas de las diferencias de anomalías entre los eventos canónicos y los Modoki para determinar el impacto asociado con cada evento en el régimen de precipitación en Colombia. Este análisis se hizo con base en la información satelital de la precipitación proveniente de la TRMM para el periodo de 1998 a 2015 y de las estaciones del IDEAM para el período de 1970 a 2015. El porcentaje de acuerdo en el signo de las anomalías de precipitación entre las bases datos también se calculó con el objeto de determinar el grado de correspondencia entre las bases de datos satelitales y las estaciones in situ. 
Tabla 1. Estaciones de registro de la precipitación incluidas en la base de datos del IDEAM

\begin{tabular}{|c|c|c|c|c|c|c|c|c|}
\hline Estación & Departamento & Código & Longitud & Latitud & $\begin{array}{c}\text { Altura } \\
\text { (msnm.) }\end{array}$ & $\begin{array}{c}\text { Precipitación } \\
(\mathbf{m m})^{*}\end{array}$ & $\begin{array}{c}\text { Fecha de } \\
\text { instalación }\end{array}$ & $\begin{array}{c}\text { Porcentaje } \\
\text { de datos }\end{array}$ \\
\hline Vásquez Cobo & Amazonas & 48015010 & $-69,56$ & $-4,11$ & 84 & 154155,8 & 1968 & 100,0 \\
\hline Santa Teresa & Antioquia & 11110010 & $-76,08$ & 6,45 & 1.370 & 121208,3 & 1969 & 99,6 \\
\hline Buchado & Antioquia & 11060010 & $-76,46$ & 6,25 & 18 & 238813,7 & 1969 & 99,8 \\
\hline E. Cortissoz & Atlántico & 29045020 & $-74,46$ & 10,53 & 14 & 39858,9 & 1940 & 99,5 \\
\hline Arauca & Arauca & 37055010 & $-70,44$ & 7,04 & 128 & 82447,5 & 1950 & 100,0 \\
\hline Rafael Núñez & Bolívar & 14015020 & $-75,30$ & 10,26 & 2 & 49441,7 & 1941 & 100,0 \\
\hline Guacamayo & Bolívar & 25020030 & $-74,30$ & 8,43 & 54 & 210004,8 & 1966 & 100,0 \\
\hline El Níspero & Bolívar & 29030310 & $-75,25$ & 9,56 & 10 & 69646,7 & 1969 & 99,6 \\
\hline Surbata Bonza & Boyacá & 24035120 & $-73,04$ & 5,48 & 2.485 & 40634,3 & 1944 & 99,8 \\
\hline Chinavita & Boyacá & 35070070 & $-73,21$ & 5,09 & 1.900 & 70567,5 & 1955 & 99,5 \\
\hline La María & Caldas & 26160160 & $-75,34$ & 5,35 & 704 & 115138,8 & 1962 & 100,0 \\
\hline La Nubia & Caldas & 26155110 & $-75,28$ & 5,01 & 2.058 & 71451,6 & 1968 & 99,5 \\
\hline Río Palo & Cauca & 26040310 & $-76,22$ & 3,04 & 1.100 & 103824,2 & 1964 & 99,6 \\
\hline Gartunduaga & Caquetá & 44035020 & $-75,33$ & 1,35 & 244 & 171339,5 & 1969 & 99,8 \\
\hline Pto. Mosquito & Cesar & 23210010 & $-73,45$ & 8,11 & 90 & 82513,9 & 1960 & 100,0 \\
\hline Tamalameque & Cesar & 25020090 & $-73,48$ & 8,51 & 20 & 92719,6 & 1960 & 99,5 \\
\hline Rincón Hondo & Cesar & 25020260 & $-73,29$ & 9,23 & 100 & 113376,3 & 1963 & 99,8 \\
\hline El Carano & Chocó & 11045010 & $-76,38$ & 5,41 & 53 & 374290,4 & 1947 & 99,8 \\
\hline Carmen de Atrato & Chocó & 11020010 & $-76,08$ & 5,54 & 1.850 & 120046,8 & 1958 & 99,6 \\
\hline Tibaitatá & Cundinamarca & 21205420 & $-74,12$ & 4,41 & 2.543 & 31373 & 1954 & 99,8 \\
\hline Cañaverales & La Guajira & 15060080 & $-72,50$ & 10,45 & 230 & 51097,5 & 1963 & 100,0 \\
\hline La Argentina & Huila & 21050060 & $-75,58$ & 2,11 & 1.500 & 70566,2 & 1958 & 99,8 \\
\hline Mesa Redonda & Huila & 21110160 & $-75,11$ & 3,07 & 500 & 52114,1 & 1969 & 99,6 \\
\hline Simón Bolívar & Magdalena & 15015050 & $-74,13$ & 11,07 & 4 & 24556,9 & 1962 & 100,0 \\
\hline Ariguaní & Magdalena & 25021250 & $-74,05$ & 9,54 & 120 & 63277 & 1953 & 100,0 \\
\hline Antonio Nariño & Nariño & 52045020 & $-77,17$ & 1,23 & 1.816 & 58152,9 & 1957 & 100,0 \\
\hline Cumbal & Nariño & 52050110 & $-77,47$ & 0,54 & 3.092 & 44214,5 & 1958 & 100,0 \\
\hline San Luis & Nariño & 52055010 & $-77,40$ & 0,51 & 2.961 & 41861,8 & 1941 & 100,0 \\
\hline Cucutilla & Norte de Santander & 16020080 & $-72,46$ & 7,32 & 1.280 & 86749,4 & 1955 & 100,0 \\
\hline Labateca & Norte de Santander & 37010010 & $-72,30$ & 7,17 & 1.560 & 47205,3 & 1955 & 99,6 \\
\hline El Edén & Quindío & 26125060 & $-75,45$ & 4,27 & 1.229 & 99890 & 1949 & 99,5 \\
\hline Matecaña & Risaralda & 26135040 & $-75,44$ & 4,48 & 1.342 & 109411,5 & 1947 & 99,8 \\
\hline Sesquicentena & San Andrés & 17015010 & $-81,42$ & 12,35 & 1 & 88652,7 & 1958 & 99,6 \\
\hline Jesús María & Santander & 24010210 & $-73,46$ & 5,52 & 1.920 & 129220,4 & 1958 & 100,0 \\
\hline San Vicente & Santander & 24050060 & $-73,24$ & 6,52 & 721 & 91762,3 & 1958 & 100,0 \\
\hline Purificación & Tolima & 21130010 & $-74,51$ & 3,51 & 345 & 87749,4 & 1958 & 99,5 \\
\hline Prado & Tolima & 21160040 & $-74,48$ & 3,44 & 360 & 104980,8 & 1959 & 99,6 \\
\hline Cabuyal & Valle & 26060020 & $-76,20$ & 3,19 & 1.000 & 58655,1 & 1953 & 99,8 \\
\hline
\end{tabular}

*Precipitación acumulada en el período 1970-2015 
Correlaciones rezagadas. Con el propósito de establecer las anomalías de la precipitación asociadas con los diferentes eventos de ENOS y el tiempo de respuesta del régimen de precipitación durante las diferentes fases de este fenómeno, se analizaron las correlaciones simultáneas y rezagadas por mes entre los índices del Niño 3, el Niño 3.4, el Niño 4, el ONI y el EMI, y las anomalías de precipitación calculadas a partir de la información suministrada por el IDEAM y los datos satelitales de la TRMM, es decir, se analizaron las correlaciones de desfase desde los 0 meses hasta los 11 . Además, se calcularon individualmente las correlaciones simultáneas y rezagadas en varias de las estaciones de la base de datos del IDEAM para conocer el comportamiento de la precipitación ante la presencia de episodios de El Niño, El Niño Modoki, La Niña y La Niña Modoki en áreas más localizadas. La significación de estas correlaciones se calculó a partir de una prueba t-Student de dos colas para un nivel de significación de $5 \%$.

\section{Resultados y discusión}

Presencia de eventos de El Niño, La Niña, El Niño Modoki y La Niña Modoki. En la Tabla 2 se muestran los trimestres en que se presentaron los eventos de El Niño, El Niño Modoki, La Niña y La Niña Modoki. Hubo eventos de El Niño en seis ocasiones durante los trimestres de diciembre a febrero, de marzo a mayo, y de junio a agosto, y en ocho ocasiones en el trimestre de septiembre a noviembre. En este caso, se pudieron observar ciclos de 12 meses de incidencia de los eventos de El Niño como el ocurrido a partir del trimestre de septiembre a noviembre de 1982 hasta el de junio a agosto de 1983, el ocurrido a partir del trimestre de diciembre a febrero de 1987 hasta el de septiembre a noviembre de ese mismo año, y el transcurrido desde el trimestre de junio a agosto de 1997 hasta el de marzo a mayo de 1998 , lo cual coincide con lo reportado en los estudios de Grimm \& Tedeschi (2009) y CórdobaMachado, et al. (2015).

Tabla 2. Años en los que se presentaron los fenómenos de El Niño Canónico, El Niño Modoki, La Niña Canónica y La Niña Modoki

\begin{tabular}{|c|c|c|c|c|c|c|c|}
\hline $\begin{array}{l}\text { Diciembre a } \\
\text { febrero }\end{array}$ & $\begin{array}{c}\text { Marzo a } \\
\text { mayo }\end{array}$ & $\begin{array}{c}\text { Junio a } \\
\text { agosto }\end{array}$ & $\begin{array}{c}\text { Septiembre a } \\
\text { noviembre }\end{array}$ & $\begin{array}{l}\text { Diciembre a } \\
\text { febrero }\end{array}$ & $\begin{array}{c}\text { Marzo a } \\
\text { mayo }\end{array}$ & $\begin{array}{c}\text { Junio a } \\
\text { agosto }\end{array}$ & $\begin{array}{c}\text { Septiembre a } \\
\text { noviembre }\end{array}$ \\
\hline \multicolumn{4}{|c|}{ El Niño Canónico } & \multicolumn{4}{|c|}{ La Niña Canónica } \\
\hline 1973 & 1983 & 1972 & 1972 & 1971 & 1970 & 1970 & 1970 \\
\hline 1983 & 1987 & 1983 & 1982 & 1974 & 1971 & 1971 & 1971 \\
\hline 1987 & 1992 & 1987 & 1987 & 1976 & 1974 & 1973 & 1973 \\
\hline 1992 & 1993 & 1997 & 1997 & 1989 & 1975 & 1975 & 1975 \\
\hline 1998 & 1998 & 2009 & 2002 & 2000 & 1985 & 1988 & 1988 \\
\hline \multirow[t]{3}{*}{2010} & 2015 & 2015 & 2006 & 2008 & 1989 & 1999 & 1999 \\
\hline & & & 2009 & & 2007 & 2000 & 2007 \\
\hline & & & 2015 & & & 2007 & 2010 \\
\hline \multicolumn{4}{|c|}{ El Niño Modoki } & \multicolumn{4}{|c|}{ La Niña Modoki } \\
\hline 1978 & 1982 & 1982 & 1977 & 1974 & 1984 & 1984 & 1973 \\
\hline 1987 & 1991 & 1987 & 1982 & 1989 & 1989 & 1988 & 1975 \\
\hline 1991 & 1992 & 1991 & 1986 & 1999 & 1999 & 1989 & 1983 \\
\hline 1992 & 1993 & 1993 & 1987 & 2000 & 2000 & 1998 & 1988 \\
\hline 1993 & 1995 & 1994 & 1990 & 2006 & 2008 & 1999 & 1995 \\
\hline 1995 & 1997 & 2002 & 1991 & 2008 & 2011 & 2000 & 1998 \\
\hline 2003 & 2003 & 2004 & 1993 & 2009 & & 2008 & 1999 \\
\hline 2005 & 2015 & 2015 & 1994 & 2011 & & 2010 & 2000 \\
\hline 2010 & & & 2002 & 2012 & & & 2008 \\
\hline \multirow[t]{4}{*}{2015} & & & 2004 & & & & 2010 \\
\hline & & & 2006 & & & & 2011 \\
\hline & & & 2009 & & & & \\
\hline & & & 2015 & & & & \\
\hline
\end{tabular}


En el caso de los eventos de El Niño Modoki, se encontró que ocurrieron en diez ocasiones en los trimestres de diciembre a febrero; en ocho ocasiones en los trimestres de marzo a mayo y de junio a agosto, y en 13 ocasiones en el trimestre de septiembre a noviembre, en los cuales el EMI presentó un valor superior a 0,7. En cuanto a los eventos de El Niño Modoki, se presentaron con un ciclo de más de 12 meses entre el trimestre de diciembre a febrero de 1991 hasta el de marzo a mayo de 1992, y con dos ciclos de 12 meses durante los años 1993 y 2015. La presencia de más episodios de El Niño Modoki que de El Niño durante el período de 1970 a 2015 concuerda con los hallazgos de Ashok, et al. (2007), Yeh, et al. (2009), y Roxy, et al. (2013), quienes encontraron que los eventos Modoki ocurrieron a partir de los años 90 con mayor frecuencia, lo cual probablemente está asociado con el calentamiento global.

En la Tabla 2 se presentan los trimestres de incidencia de los eventos de La Niña, es decir, los períodos en los cuales las anomalías normalizadas de la TSM están por debajo de $-1,0$ en la región del Niño 3. Los eventos de La Niña se presentaron en seis ocasiones en los trimestres de diciembre a febrero; en siete ocasiones en el de marzo a mayo, y en ocho ocasiones en el de junio a agosto y en el de septiembre a noviembre. Entre los ciclos observados para este tipo de evento estuvo el ocurrido desde el trimestre de marzo a mayo de 1970 hasta el de diciembre a febrero de 1971; los datos del ONI reflejan un comportamiento similar de este evento, ya que se observó un ciclo de 18 meses para el evento de La Niña transcurrido entre el trimestre de junio a agosto de 1970 hasta el de diciembre a febrero de 1971. Otros ciclos de menor duración en los cuales se presentó esta fase del ENOS fueron el trimestre de junio a agosto de 1973 hasta el de marzo a mayo de 1974; el de marzo a mayo de 1975 hasta el de diciembre a febrero de 1976; el de junio a agosto de 1988 hasta el de marzo a mayo de 1989, y el de marzo a mayo del 2007 hasta el de diciembre a febrero de 2008.

El evento de La Niña Modoki ocurrió en nueve ocasiones en el trimestre de diciembre a febrero; en seis ocasiones en el trimestre de marzo a mayo; en ocho ocasiones en el de junio a agosto, y en 11 ocasiones en el de septiembre a noviembre, durante los cuales el EMI presentó un valor inferior a -0,7. A partir del trimestre de junio a agosto de 1988 hasta el mismo trimestre de 1989, se observó uno de los ciclos de este evento durante el período de 1970 a 2015; otro ciclo comprendió el período entre el trimestre de junio a agosto de 1998 hasta el de diciembre a febrero del 2000, y un tercer ciclo se observó entre el trimestre de diciembre a febrero de 2008 hasta el mismo trimestre de 2009. Estos períodos de incidencia del evento de La Niña Modoki fueron bastante similares a los reportados por Córdoba-Machado, et al. (2015) en su estudio, en el cual pudieron observar amplios períodos en los que ocurrió esta fase del ENOS durante esos mismos años. En el presente estudio se reporta el último ciclo de 12 meses en el que ocurrió el evento de La Niña Modoki entre el trimestre de junio a agosto de 2010 hasta el de marzo a mayo de 2011.
A continuación se analizan las anomalías de precipitación en Colombia asociadas con los diferentes eventos del ENOS para los trimestres de diciembre a febrero y de junio a agosto, temporadas en las que se observaron los mayores y los menores efectos, respectivamente.

Anomalías en la precipitación durante el trimestre de diciembre a febrero. En la Figura 1 se presentan las anomalías de precipitación registradas en el trimestre de diciembre a febrero para cada tipo de evento del fenómeno de ENOS con base en los datos obtenidos de la TRMM durante el período de 1998 a 2015 y la información suministrada por el IDEAM durante el período de 1970 a 2015. Los datos de la TRMM evidencian que durante los eventos de El Niño hubo marcadas anomalías negativas en el sur y el occidente del país, en tanto que en el norte, oriente y parte del centro de Colombia se presentaron anomalías negativas con menor intensidad. Por otro lado, los datos registrados en las estaciones del IDEAM reflejaron anomalías de precipitación negativas en gran parte del territorio, con excepción de algunas estaciones ubicadas hacia el occidente, en el departamento de Chocó, de otra estación en el suroccidente y de otra en el sur del departamento de Amazonas, las cuales registraron valores positivos. La información obtenida de las estaciones evidenció que la zona norte de país presentó homogeneidad en la magnitud de las anomalías negativas, en tanto que en la zona andina los valores de magnitud fueron más variables y en algunas estaciones se pudo comprobar, incluso, que el régimen de precipitación puede no verse afectado por este evento, pues se registraron valores de anomalías cercanos a 0 . El porcentaje de acuerdo en el signo de las anomalías de precipitación entre la información de la TRMM y la del IDEAM fue de $71 \%$.

Durante los eventos de El Niño Modoki, las anomalías negativas de precipitación se presentaron en las áreas central, oeste y norte de Colombia, siendo más débiles que las anomalías ocurridas durante el evento de El Niño, lo que coincide con lo reportado por Tedeschi, et al. (2015). Este patrón se observó tanto en los datos de la TRMM como en la información del IDEAM. Para el evento de El Niño Modoki, las bases de datos registraron un porcentaje de acuerdo en el signo de las anomalías de precipitación de 84,2\%. Hacia el sur del país, los valores de las anomalías fueron positivos, aunque en algunas estaciones se registraron valores de anomalías positivas en el occidente y el suroccidente. Durante los eventos de El Niño predominaron condiciones más secas de lo normal en todo el país, en tanto que durante los de El Niño Modoki el comportamiento del régimen de precipitación ante las anomalías de la TSM en el Pacífico tropical no fue generalizado en cuanto al signo. Las diferencias en los efectos generados por El Niño Canónico y El Niño Modoki fueron más evidentes en los datos satelitales que en los de las estaciones del IDEAM. La mayor diferencia se encontró en la zona del suroriente y el occidente de Colombia con base en la información de la TRMM, en tanto que en el área del norte y parte del centro del 

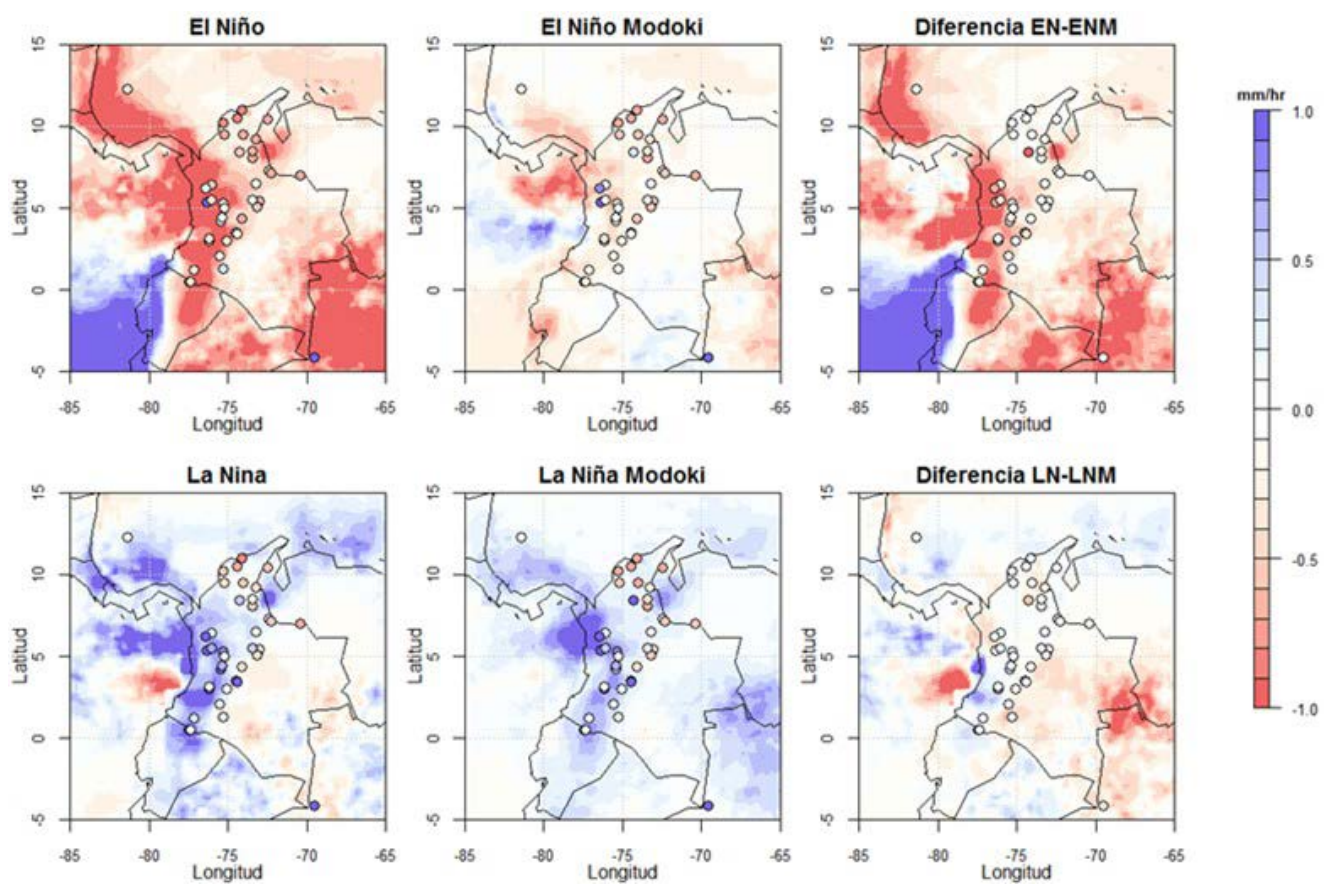

Figura 1. Anomalías de precipitación durante los eventos de El Niño, El Niño Modoki, La Niña y La Niña Modoki y diferencia entre los eventos canónicos y los Modoki para el trimestre de diciembre a febrero. En colores se presentan las anomalías obtenidas de los registros de la TRMM y con círculos, aquellas correspondientes a los datos suministrados por el IDEAM.

territorio, el impacto de estos dos eventos en la precipitación varió poco en los dos tipos de datos. En algunas estaciones del IDEAM se evidenciaron diferencias significativas en los valores negativos entre los dos eventos de El Niño, lo que quiere decir que en ellas la precipitación durante El Niño Modoki fue mayor que durante El Niño y evidencia una atenuación en el efecto de la fase de El Niño en la precipitación de la región. Dos de esas estaciones están en el oeste y otra, más al norte. En el resto de estaciones las diferencias fueron casi nulas.

Los eventos de La Niña se han asociado con anomalías positivas en la precipitación en la región norte de Suramérica en diferentes periodos (Tedeschi, et al., 2012). Sin embargo, si se hace una análisis más local, puede observarse que para el caso de Colombia los datos satelitales evidencian anomalías negativas de precipitación en el centro, oriente y sur del país durante el trimistre de diciembre, enero y febrero; además, las anomalías registradas en las estaciones in situ presentaron valores negativos en el norte, con excepción de una estación. Tanto con los datos de la TRMM como con los del IDEAM reflejaron las anomalías positivas asociadas con eventos de La Niña en el occidente y suroccidente del país. En cuanto al fenómeno de La Niña Modoki, según la información de la TRMM ocurrió un aumento de la precipitación en casi todo el territorio, con claras anomalías positivas hacia el occidente y el suroccidente, lo que concuerda con los datos del IDEAM. Hacia el norte, las estaciones in situ registraron anomalías negativas de precipitación con valores muy similares a los de La Niña, como puede observarse en el mapa que muestra las diferencias. Los datos satelitales también evidenciaron que en gran parte del territorio colombiano hubo mayor precipitación durante los eventos de La Niña Modoki que durante La Niña, con excepción de algunas zonas en el norte y el suroccidente del país. El porcentaje de acuerdo en el signo de las anomalías de precipitación entre los datos de la TRMM y la información suministrada por el IDEAM fue de $68,4 \%$ para los episodios de La Niña y de $57,9 \%$ para los del La Niña Modoki.

Anomalías de precipitación durante el trimestre de junio a agosto. En la Figura 2 se presentan los mapas de las anomalías de precipitación para cada evento del fenómeno ENOS durante el trimestre de junio a agosto. Según los datos de la TRMM, durante la incidencia de los eventos de El Niño hubo anomalías negativas en el norte, occidente y centro del país, en tanto que hubo claras anomalías positivas en la parte sur y oriente. En ese trimestre, los efectos de El Niño no tendieron a generar una sequía generalizada, ya que el impacto varío dependiendo de la región. La mayoría de los datos del IDEAM exhibieron anomalías negativas, especialmente en el área andina y en el norte, lo que concuerda con los datos de la TRMM. En algunas estaciones se registraron anomalías positivas en algunas partes del territorio colombiano, lo cual coincidió con la información satelital en las estaciones ubicadas en el nororiente y en el suroccidente. El porcentaje de acuerdo en el signo de las anomalías de precipitación entre la información in situ y la satelital fue de $78,9 \%$. 

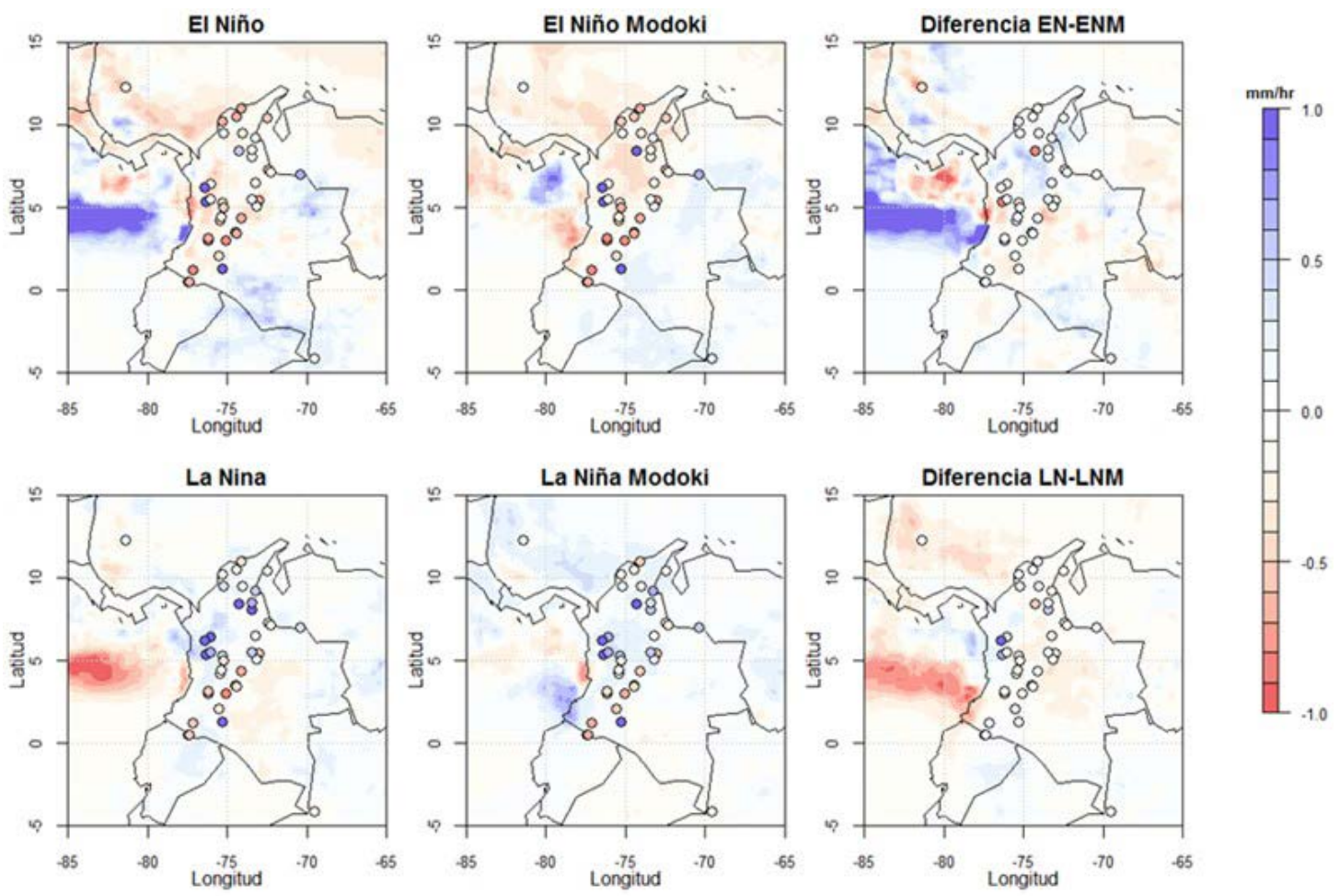

Figura 2. Anomalías de precipitación durante los eventos de El Niño, El Niño Modoki, La Niña y La Niña Modoki y diferencia entre los eventos canónicos y los Modoki para el trimestre de junio a agosto

En el mapa de la precipitación durante los eventos de El Niño Modoki, los datos de la TRMM evidenciaron claras anomalías negativas en el norte y parte del occidente de Colombia, lo que también ocurrió en la gran mayoría de las estaciones del IDEAM ubicadas en esas zonas; en el oriente, el sur y el suroccidente se observaron anomalías positivas con los datos de las dos fuentes, aunque menores a las registradas en el estudio de Silva (2012) en las regiones del sur de Colombia, norte de Perú y noroccidente de Brasil. El porcentaje de acuerdo de la información de las estaciones in situ y la de la TRMM durante este evento fue de 89,5\%. Las estaciones ubicadas en el norte y occidente del país, cuya información difirió del patrón de los datos satelitales, evidenciaron anomalías positivas de precipitación. Al igual que en el evento de El Niño, la fase de El Niño Modoki no mostró una tendencia al aumento o disminución de la precipitación generalizada en el país, sino que su impacto varió según la zona.

La ilustración de las diferencias entre las anomalías de precipitación generadas por los episodios cálidos y las anomalías en la TSM en el este y centro del océano Pacífico tropical demuestra que durante el fenómeno de El Niño Modoki hubo una mayor disminución de la lluvia que durante El Niño en la zona norte del país, como pudo verse en la información de la TRMM y de algunas estaciones del IDEAM; según la TRMM, durante los eventos de El Niño la precipitación fue mayor hacia el sur que durante El Niño Modoki; en parte del suroccidente se pudo apreciar que durante El Niño hubo disminución de la precipitación en tanto que durante El Niño Modoki hubo un aumento. En los registros de algunas estaciones ubicadas en el área andina se evidenció que hubo una mayor disminución de la precipitación durante El Niño Modoki que durante El Niño, pero en el suroccidente no se observó una clara diferencia entre un evento y otro.

A diferencia de los eventos de El Niño y El Niño Modoki, durante los cuales hubo claras anomalías negativas hacia el norte, occidente y parte del centro según los datos de la TRMM, los mapas de los eventos de La Niña y La Niña Modoki muestran anomalías positivas en esas áreas, así como hacia el oriente del país. Hubo un mayor número de estaciones del IDEAM que registraron anomalías positivas durante estos eventos que durante El Niño y El Niño Modoki, aunque el patrón de disminución o de aumento de las lluvias a lo largo del territorio tendió a ser muy similar durante los eventos de La Niña y La Niña Modoki según la información in situ y la satelital, como puede observarse en los tres mapas inferiores de la Figura 2. En el mapa de las diferencias se observan algunas estaciones con valores positivos, lo que quiere decir que durante los episodios de $\mathrm{La}$ Niña hubo mayor precipitación, pero en algunas estaciones del suroccidente con diferencias positivas se evidenció que durante La Niña Modoki hubo una mayor disminución de las lluvias. Por otro lado, la información de la TRMM permitió ver que en el norte y el centro de Colombia hubo una mayor precipitación durante los eventos de La Niña Modoki que 
durante los eventos de La Niña, lo cual concuerda con parte de la información proveniente de las estaciones ubicadas en esas áreas.

Correlación simultánea. En la Figura 3 se observa que cada uno de los índices relacionados con el ENOS reflejó correlaciones negativas significativas con las anomalías de precipitación en el área andina, en parte del occidente, el norte y el nororiente de Colombia tanto en la información de la TRMM como en la del IDEAM. El índice del Niño 4 y el EMI evidenciaron una pequeña área de correlaciones positivas hacia el suroccidente y el occidente del país, lo que puede verse en los datos de la TRMM. Por su parte, la mayoría de las estaciones del IDEAM registraron correlaciones negativas, pues en el $84,2 \%$ de las estaciones se registraron valores distintos de cero para los índices del Niño 3, el Niño 3.4 y el ONI, mientras que el índice EMI evidenció correlaciones negativas significativas en el $71 \%$ de las estaciones y el Niño 3.4 en el 84,2 \% de ellas, lo cual resalta la relación inversa existente entre las temperaturas del Pacífico este y central y la precipitación en Colombia: ante un calentamiento (o enfriamiento) de esta región del Pacífico tropical, se registra una reducción (o incremento) de la precipitación en el país. Sin embargo, el análisis de las correlaciones rezagadas de 1 a 11 meses reveló que este efecto no fue igual en todas las regiones del país (Figuras 4 y 5 ).

Correlaciones rezagadas. Para describir la asociación existente entre las anomalías de precipitación en Colombia y los distintos índices considerados para describir los eventos canónicos y Modoki del ENOS, se analizaron las correlaciones significativas con un desfase de 0 hasta 11 meses según la información suministrada por el IDEAM y la base de datos satelitales de la TRMM. Los rezagos considerados son tales que las anomalías de la TSM anteceden las anomalías en la precipitación, lo que también permite observar el tiempo de respuesta del régimen de precipitaciones ante la presencia de los distintos eventos del ENOS. Aquí se presentan los mapas de las correlaciones rezagadas de cero a seis meses, es decir el tiempo durante el cual se observan áreas de Colombia bajo la influencia de estos eventos. Dichas correlaciones se establecieron con un nivel de confianza de $95 \%$.

Correlación rezagada de seis meses. La Figura 4 muestra las correlaciones significativas entre los índices del ENOS considerados y las anomalías de precipitación con un desfase de seis meses. En el mapa correspondiente al índice del Niño 3 se ve un área de correlaciones significativas en el suroccidente del país según la información de la TRMM, en tanto que se observan algunas estaciones del IDEAM con valores de correlación diferentes de cero en el occidente, el suroccidente y el nororiente, las cuales representan el 34,2 \% del total de las estaciones. Los mapas de los índices del Niño 3.4 y el ONI exhiben correlaciones negativas significativas en una pequeña área de los Andes según los datos de la TRMM y los del IDEAM, aunque en algunas estaciones se evidenciaron correlaciones significativas hacia el nororiente del país en el índice del Niño 3.4. Por ello, el porcentaje de estaciones con correlaciones negativas significativas para
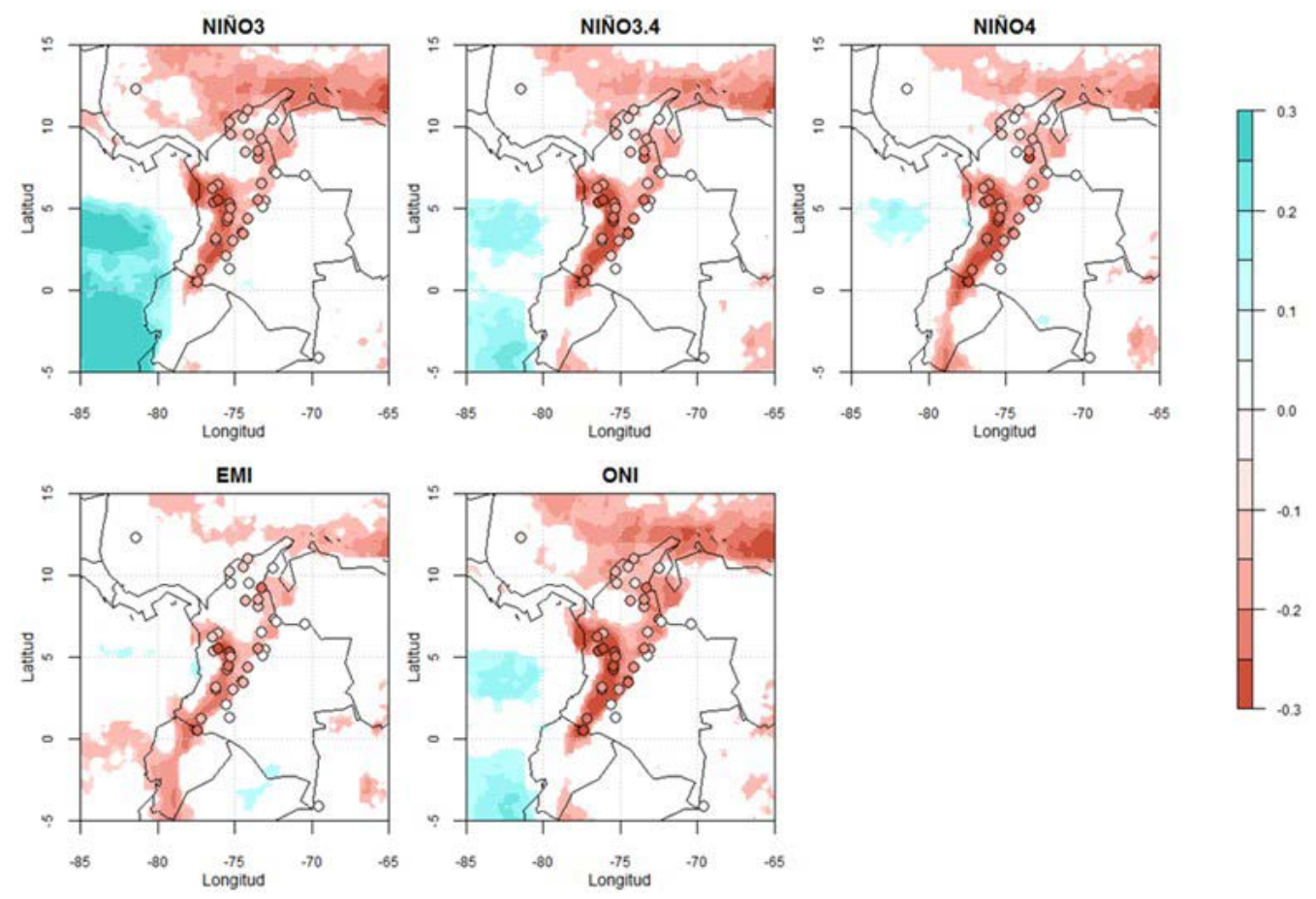

Figura 3. Correlación simultánea entre los índices del ENOS y las anomalías en la precipitación. Solo se presentan los valores estadísticamente significativos para un nivel de confianza del $95 \%$. 

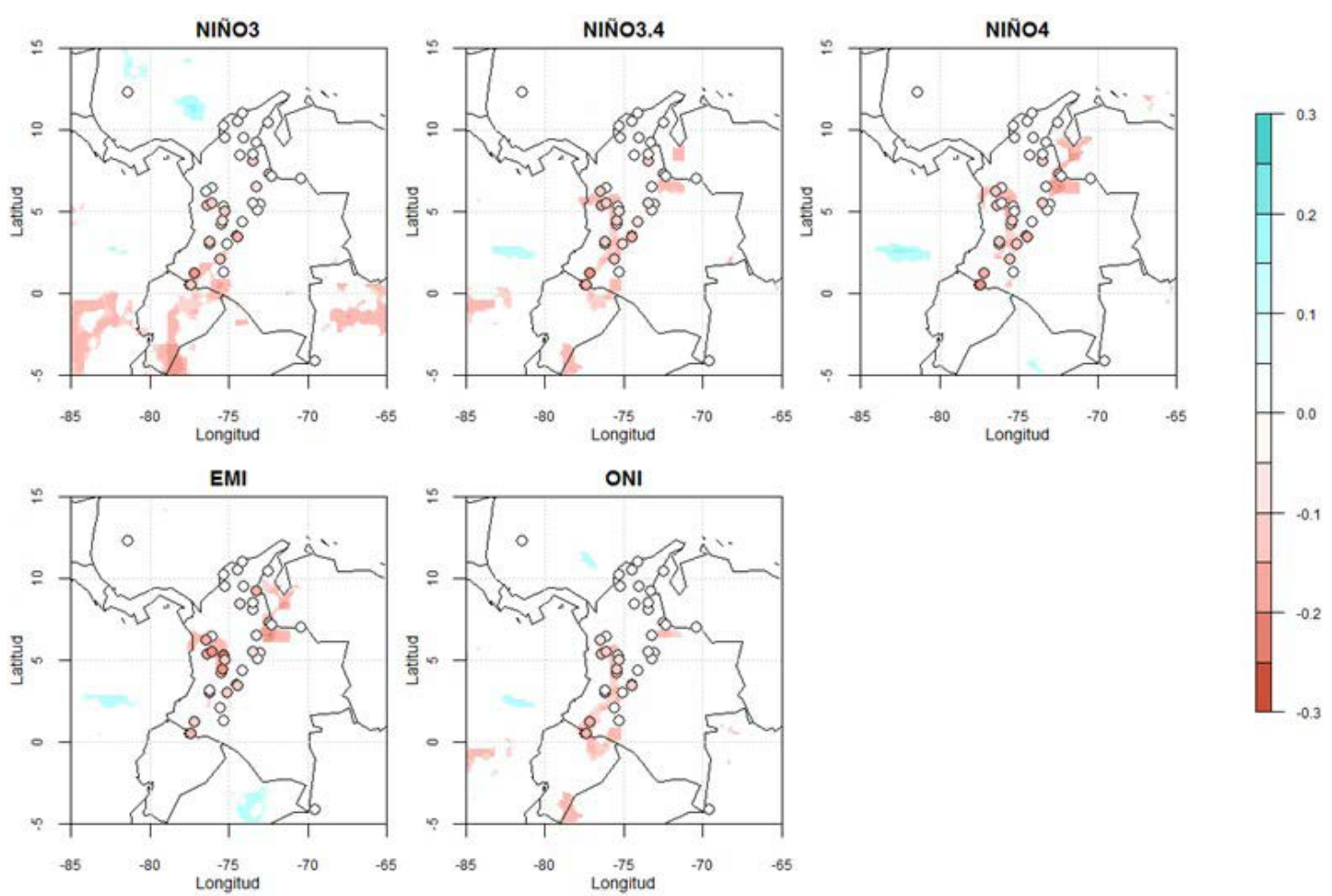

Figura 4. Correlación simultánea entre los índices del ENOS y las anomalías en la precipitación con una rezago de seis meses. Solo se presentan los valores estadísticamente significativos para un nivel de confianza del $95 \%$. Las anomalías en la TSM anteceden las anomalías en la precipitación.

este índice fue de $42 \%$, en tanto que según el índice del Niño 3 el porcentaje de estaciones fue de 28,9\%. Por último, para los índices del Niño 4 y el EMI los mapas muestran correlaciones negativas significativas en el occidente y el nororiente según la información de la TRMM y en algunas estaciones del IDEAM ubicadas en esas áreas. En los mapas de los índices que describen eventos Modoki también se observan estaciones del IDEAM con valores de correlaciones negativas significativas hacia el suroccidente y nororiente; el porcentaje de estaciones con este comportamiento fue de $34,2 \%$ para el índice del Niño 4 y de 42,1 \% para el EMI.

Correlogramas de los datos de las estaciones del IDEAM. En la Figura 5 se presentan los correlogramas de los diferentes índices empleados y las anomalías de precipitación con rezagos de cero hasta 11 meses en cuatro estaciones del IDEAM. Hacia el noreste de Colombia se presentaron las correlaciones más bajas, con un valor aproximado de $-0,2$, y con el tiempo de respuesta más corto, siendo hasta de cuatro meses para los índices del Niño 3, el ONI y el Niño 4, y de cinco meses para los índices del Niño 3.4 y el EMI. En las estaciones del suroccidente y el occidente se presentaron los valores más altos de correlación, aproximadamente de -0,25. En estas estaciones el tiempo de respuesta de las anomalías en la precipitación fue de seis, siete, ocho y hasta nueve meses después de presentarse las anomalías en la TSM asociadas al ENOS. La estación situada en el centro del país registró tiempos de respuesta con cinco a seis meses de rezago. En esta zona la respuesta de la precipitación medida con los índices del Niño 3 y el ONI fue similar, en tanto que con los índices del Niño 4 y el EMI se registró el mismo tiempo de respuesta, pero la magnitud de las correlaciones varió, con valores de $-0,2$ con el EMI y de -0,25 con el Niño 4.

\section{Conclusiones}

Al clasificar los trimestres en los que ocurrieron eventos canónicos y Modoki del ENOS durante el período de 1970 a 2015, se encontró que los episodios de El Niño Modoki y La Niña Modoki fueron más frecuente que los episodios de El Niño y La Niña. Se observó que en el trimestre de septiembre a noviembre ocurrieron ocho eventos de El Niño y La Niña, 13 de El Niño Modoki y 11 de La Niña Modoki; en el trimestre de diciembre a febrero ocurrieron seis eventos de El Niño y La Niña, diez eventos de El Niño Modoki y nueve de La Niña Modoki; en los trimestres de marzo a mayo y de junio a agosto, la diferencia entre el número de eventos fue menor, con seis episodios de El Niño y ocho de El Niño Modoki, La Niña y La Niña Modoki durante junio a agosto y seis eventos de El Niño, ocho de El Niño Modoki, siete de La Niña y seis de La Niña Modoki en el trimestre de marzo a mayo.

La mayor influencia de los eventos de ENOS en el régimen de precipitación en Colombia se observó durante el trimestre de diciembre a febrero, en tanto que la menor 


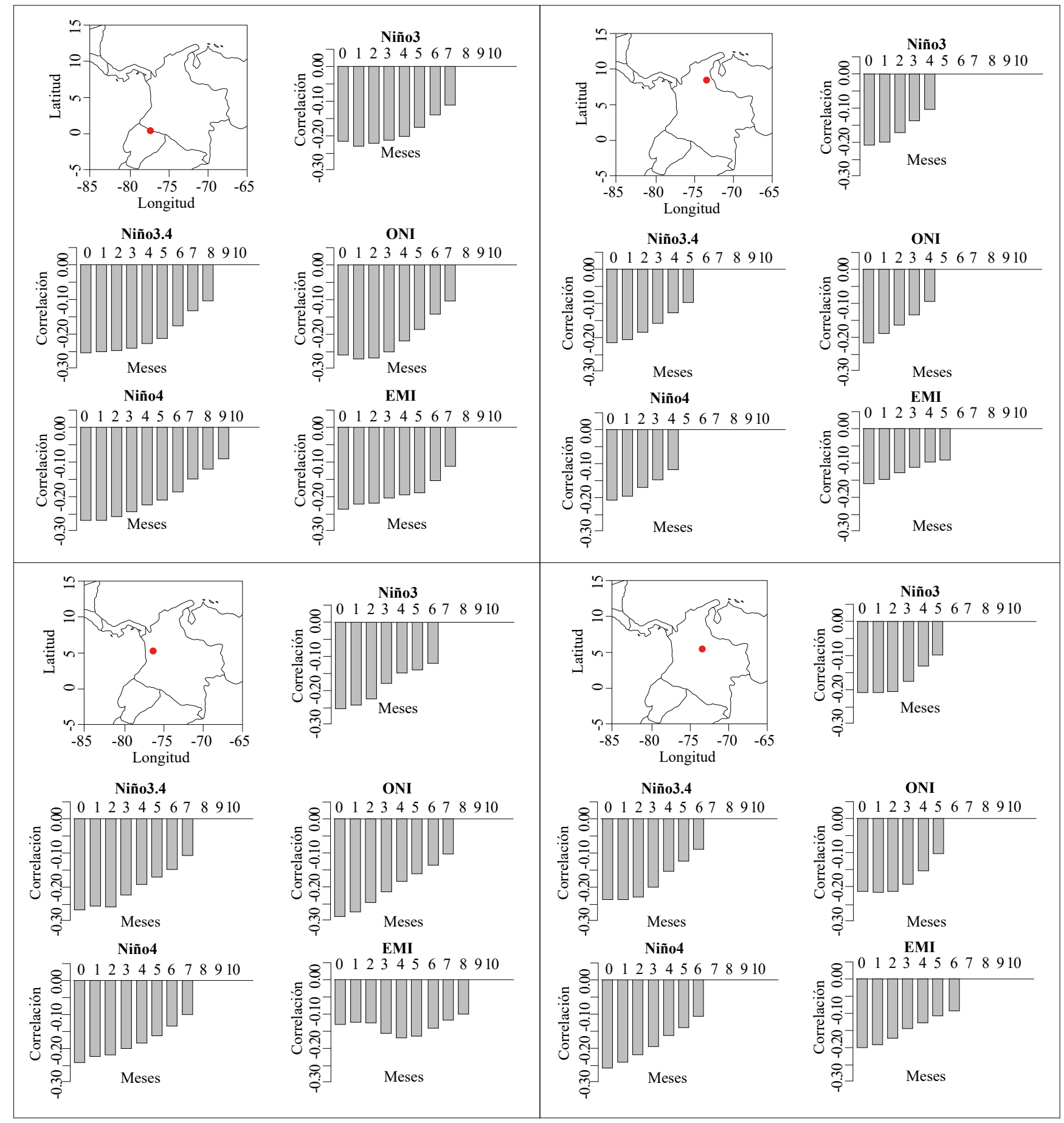

Figura 5. Correlogramas de los distintos índices considerados para los eventos de ENOS y las anomalías en la precipitación con rezagos de cero hasta 11 meses en cuatro estaciones en diferentes regiones de Colombia. Las anomalías en la TSM anteceden a las anomalías en la precipitación. En los mapas aparece la ubicación de la estación correspondiente a cada región.

influencia se encontró en las temporadas de marzo a mayo (estos datos no se presentan) y de junio a agosto, según los datos de la TRMM. Esta respuesta de las anomalías en la precipitación en Colombia durante estos eventos es razonable, pues en el trimestre de diciembre a febrero el fenómeno de ENOS se encuentra en su máximo desarrollo, en tanto que la temporada de marzo a mayo es de transición en las condiciones de la temperatura en el océano Pacífico tropical (Tedeschi, et al., 2016a).

$\mathrm{El}$ análisis del porcentaje de acuerdo en el signo de las anomalías en la precipitación entre los datos obtenidos de los registros in situ y la información satelital evidenció que 
hubo una mayor coincidencia en los eventos de El Niño que en los de La Niña. Es posible que estos porcentajes puedan afinarse a medida que se realice un mayor control de calidad de la información empleada y de los análisis de un mismo período. Sin embargo, en futuros estudios se recomienda hacer un análisis estadístico para verificar con mayor detalle la confiabilidad de las fuentes de datos.

Se sabe que el ENOS es el principal modulador de la variabilidad interanual de la precipitación en Colombia (Poveda, et al., 1998); sin embargo, la influencia de este fenómeno depende de la fase en la cual se encuentre y de la región de Colombia que se analice, pues las respuestas del régimen de precipitación ante las anomalías de la TSM no son totalmente homogéneas a lo largo del país. Se observó, por ejemplo, que según la información de TRMM, los episodios de El Niño durante el trimestre de diciembre a febrero causaron una mayor disminución de la precipitación en todo el país que los ocasionados por El Niño Modoki, en tanto que durante La Niña Modoki la precipitación fue mayor que durante La Niña en casi todo el territorio. Durante el trimestre de septiembre a noviembre la influencia de las dos fases de El Niño en la precipitación del país difirió poco, al igual que en las dos fases de $\mathrm{La}$ Niña durante el período de junio a agosto. En el trimestre de marzo a mayo, La Niña generó una mayor precipitación que La Niña Modoki en casi toda Colombia. Por otro lado, se observó una mayor disminución de la precipitación en parte del área andina y del norte del país durante El Niño Modoki que durante El Niño, en tanto que hacia el sur se presentaron anomalías positivas de precipitación durante la fase Modoki de El Niño.

El análisis de correlaciones rezagadas contribuyó a visualizar de manera más clara las regiones de Colombia cuyo régimen de precipitación se vio más influido por el ENOS durante el periodo de estudio. Se observó que la zona andina, parte del occidente y el norte de Colombia fueron las regiones en las que este fenómeno tuvo mayor impacto. Este análisis también fue útil para determinar el tiempo de respuesta de la precipitación al ENOS. Se encontró que las correlaciones significativas ocurrieron con cero a seis meses de rezago, lo que significa que las anomalías de precipitación en Colombia pueden tardar hasta seis meses en exhibir una respuesta a un evento de ENOS.

Los resultados de este trabajo aportan elementos nuevos en la comprensión de la variabilidad interanual de la hidroclimatología colombiana, pues la respuesta de la precipitación (y de los caudales) en Colombia ante el ENOS está condicionada por el patrón de calentamiento o enfriamiento que exhibe el Pacífico tropical durante las diferentes fases del fenómeno; además, esta respuesta depende de la región del país que se considere. Dichos datos son fundamentales para el pronóstico de la disponibilidad hídrica en las diferentes regiones de Colombia y para la formulación e implementación de estrategias de prevención ante eventos extremos de precipitación.

\section{Agradecimientos}

Este trabajo fue financiado en el marco de la convocatoria del Comité para el Desarrollo de la Investigación (CODI) para Trabajos de Grado 2016/2 de la Universidad de Antioquia.

\section{Contribución de los autores}

Estefanía Navarro-Monterroza procesó la información y realizó los análisis aquí presentados. Paola A. Arias y Sara C. Vieira diseñaron y dirigieron la investigación.

\section{Conflicto de interés}

Los autores manifiestan no presentar conflicto de intereses para este trabajo.

\section{Referencias}

Ashok, K., Behera, S. K., Rao, S. A., Weng, H., Yamagata, T. (2007). El Niño Modoki and its possible teleconnection. Journal Of Geophysical Research. 112: 1-27.

Ashok, K., Yamagata, T. (2009). The El Niño with a difference. Nature. 461: 481-484.

Córdoba-Machado, S., Palomino-Lemus, R., Gámiz-Fortis, S., Castro-Díez, Y., Esteban-Parra, M. (2015). Assessing the impact of El Niño Modoki on seasonal precipitation in Colombia. Global and Planerary Change. 124: 41-61.

Grimm, A. M., Tedeschi, R. G. (2009). ENSO and Extreme Rainfall Events in South America. Journal of Climate. 22: 1589-1609.

Grimm, A. M., Barros, V. R., Doyla, M. E. (2000). Climate Variability in Southern South America Associated with El Niño and La Niña Events. American Meteorological Society. 13: 35-58.

Kim, H.M., Webster, P. J. Curry, J. A. (2009). Impact of Shifting Patterns of Pacific Ocean Warming on North Atlantic Tropical Cyclones. Science. 325: 77-80.

McPhaden, M., Busalacchi, A. J., Cheney, R., Donguy, J. R., Gage, K. S., Halpern, D., Takeuchi, K. (1998). The Tropical Ocean-Global Atmosphere observing system: A decade of progress. Journal of Geophysical Research. 103: 14169-14240.

Philander, S. G. (1990). El Niño, La Niña, and the Southern Oscillation. San Diego, USA: Academic Press. p. 293.

Poveda, G., Gil, M. M., Quiceno, N. (1998). El ciclo anual de la hidrología de Colombia en relación con el ENSO y la NAO . Bulletin de l'Institut français d'études andines. 27: 721-731.

Rasmusson, E. M., Carpenter T. H. (1982). Variations in tropical sea surface temperature and surface wind fields associated with the Southern Oscillation/El Niño. Monthly Weather Review. 110: 354-384.

Ropelewski, C. F., Halpert, M. S. (1996). Quantifying Southern Oscillation-Precipitation Relationships. Journal of Climate. 9: 1043-1059.

Roxy, M., Patil, N., Aparna, K., Ashok, K. (2013). Revisiting the Indian Summer monsoon-ENSO links in the IPCC AR4 projections: A cautionary outlook. American Meteorological Society. 104: 51-60.

Tedeschi, R. G., Cavalcanti, I., Grimm, A. M. (2012). Influence of two types of ENSO on South American precipitation. International Journal of Climatology. 33: 1382-1400. 
Tedeschi, R. G., Grimm, A. M., Cavalcanti, I. (2015). Influence of Central and East ENSO on extreme events of precipitation in South America during austral spring and summer. International Journal of Climatology. 35: 2045-2064.

Tedeschi, R. G., Collins, M. (2016a). The influence of ENSO on South American precipitation during austral summer and autumn in observations and models. International Journal of Climatology. 36: 618-635.

Tedeschi, R. G., Grimm, A. M., Cavalcanti, I. (2016b). Influence of Central and East ENSO on precipitation and its extreme events in South America during austral autumn and winter. International Journal of Climatology. 36: 4797-4814.

Silva, A. (2012). El Niño and El Niño Modoki impacts on South American rainfall (Tesis de maestria). The University of New South Wales, Sydney, Australia. p. 191.
Wang, G., Hendon, H. H. (2007). Sensitivity of Australian Rainfall to Inter-El Niño Variations. American Meteorological Society. 20: 4211-4226.

Weng, H., Ashok, K., Behera, S. K., Rao, S. A., Yamagata, T. (2007). Impacts of recent El Niño Modoki on dry/wet conditions in the Pacific rim during boreal summer. Climate Dynamics. 29: 113-129.

Wyrtki, K. (1975). El Niño-The Dynamic Response of the Equatorial Pacific Ocean to Atmospheric Forcing. Journal of Physical Oceanography. 5: 572-584.

Yeh, S. W., Kug, J. S., Dewitte, B., Kwon, M. H., Kirtman, B. P., Jin, F. F. (2009). El Niño in a changing climate. Nature. 461: 511-514. 